\title{
On the origin of the Biomphalaria glabrata hemocytes
}

\author{
Samaly dos Santos Souza, Zilton A Andrade ${ }^{+}$ \\ Laboratório de Patologia Experimental, Centro de Pesquisas Gonçalo Moniz-Fiocruz, R. Valdemar Falcão 121, \\ 40295-001 Salvador, BA, Brasil
}

\begin{abstract}
A histologic, morphometric and ultrastructural study performed on Biomphalaria glabrata submitted to infection with Schistosoma mansoni miracidia failed to provide significant evidences that the so-called amebocyte-producing organ (APO) is really the central organ for hemocyte production. In infected snails no general reactive changes appeared in the APO, the mitoses were seen only occasionally, and the possibility of cellular hyperplasia was ruled out by morphometric measurements. Under the electron microscope the APO cells presented an essentially epithelial structure, without features indicative of transition toward hemocytes. On the other hand, the present findings pointed to a multicentric origin for the mollusck hemocytes, as earlier studies had indicated. Dense foci of hemocyte collections appeared sometimes around disintegrating sporocysts and cercariae in several organs and tissues of the infected snails, including a curious accumulation of such cells inside the ventricular cavity of the heart. In the heart and other sites, features suggestive of transformation of vascular space endothelial lining cells into hemocytes were apparent. To some extent, the postulated multicentric origin for B. glabrata hemocytes recapitulates earlier embryologic findings in vertebrates, when mesenchymal vascular spaces generate the circulating and phagocytic blood cells.
\end{abstract}

Key words: amebocyte - producing organ - hemocytes - Biomphalaria glabrata - Schistosoma mansoni

It is currently admitted that the defense cells of Biomphalaria glabrata - the hemocytes, which are found circulating free in hemolymph and wandering about within connective tissues, take its origin from a special organ present in a tiny area of the reno-pericardial zone. This organ has been designated "amebocyte forming organ" or APO, and it is supposed to be the counterpart of the vertebrate bone marrow (Jeong et al. 1983). When $B$. glabrata snails are facing a schistosome infection, the outstanding presence of mitoses and cellular hyperplasia within the APO is said to occur, indicating and active stage of new hemocyte production (Sullivan \& Cheng 1984, Sullivan et al. 2004). Another line of evidence that APO is indeed the central organ producing the cells of defense derives from studies with the technique of organ transplantation. It has been claimed that a Schistosoma mansoni susceptible snail may be turned resistant after receiving an APO from a resistant snail (Sullivan 1990, Sullivan \& Spence 1994).

Surprisingly, there are few data on record concerning the sequential pathological changes that may affect such an interesting and sensitive organ as the APO when $B$. glabrata is infected with $S$. mansoni. Present investigation was primarily planned as an attempt to fill this gap, however the results obtained by means of histopathological, histochemical, morphometric, and ultrastructural

\footnotetext{
Financial support: Fapesb-Pronex

+Corresponding author: zilton@cpqgm.fiocruz.br

Received 25 May 2006

Accepted 26 June 2006
}

methods applied to normal and S. mansoni-infected $B$. glabrata failed to provide convincing evidences that the APO is a central organ of hemocyte production. The findings were rather more consistent with the primitive concept that postulates a multicentric origin for B. glabrata hemocytes (Pan 1958, 1963, 1965).

\section{MATERIALS AND METHODS}

Snails - About 75 B. glabrata, measuring $11-13 \mathrm{~mm}$ in major diameter (40-60 days) were used. They were maintained with free access to appropriated feed, under controlled conditions of room temperature (around $26^{\circ} \mathrm{C}$ ).

Groups - Fifty snails were individually exposed to 20 freshly obtained $S$. mansoni miracidium, and other 25 were kept under the same conditions, but not infected. Ten infected and 5 non-infected snails were killed on the 1st, 5th, 15 th, 35th, and 45th days following the exposure, to be submitted to microscopic examination.

Procedures - Under partial anesthesia with menthol, the snails had their shells carefully crushed between two glass slides. The soft parts were cleaned from the shell debris and fixed in 10th, $\mathrm{pH}$ 7.2-buffered formalin, for at least $24 \mathrm{~h}$ before processed for paraffin inclusion and sectioning. The slides were routinely stained with hematoxilyn and eosin. Other sections were submitted to histochemical treatment as detailed below.

Other fixed snails had their renal-pericardial region dissected out with fine forceps and under a dissecting microscope. The removed part was embedded in paraffin separately, for the obtaining of multiple or serial sections.

Morphometry - The APO present in 6 to 8 randomly sections per snail was measured in size and length by means of an automatic morphometric system analyzer (Axioskop 2-Zeiss), calibrated for an amplification of $10 \times$ 40 and a $\mu^{2}$ area. 
Hemocyte count in lymph - Lymph was collect with a siliconized automatic pipette. A $10 \%$ solution of lymph in neutral red was placed in a Naubauer chamber and observed under an optical microscope at $10 \times 40$ magnification. Hemocytes present in 4 randomly selected fields were counted.

Electron microscopy - Tiny fragments taken from the reno-pericardial region, from infected and non-infected snails, were immediately fixed in Karnovisk fluid during 2 $\mathrm{h}$ at room temperature, followed by rapid washings in 0.2 $\mathrm{M}$ solution of cacodylate buffer, $\mathrm{pH}$ 7.4. Post-fixation was processed by immersion into a solution of $2 \%$ osmium tetroxide in $0.2 \%$ glutaraldehyde in cacodylate buffer $(1: 1)$ during $1 \mathrm{~h}$ at room temperature, and under vacum. Fragments were dehydrated in graded concentrations of acetone and embedded in Poly-bed 812 (Embedding Media Polysciences, IVC). Selected ultrathin sections $(50-70 \mathrm{~nm})$ were made with a Reichert (Ultratome Supernova Leica) ultramicrotome and mounted on uncoated copper grids, contrasted with uranyl acetate and lead citrate. Specimens were examined in a transmission Zeiss EM-9 electron microscope, which was operated at an acceleration voltage of $50 \mathrm{kV}$.

Histochemistry - Silver nucleolar organizer region $(A g N O R)$ - Deparaffinized sections were submitted to treatment with two drops of a $2 \%$ gelatin solution and to four drops of $1 \%$ silver nitrate solution. The wet sections were covered with cover slips and placed in an oven under the temperature of $60^{\circ} \mathrm{C}$ for 3-4 min up to the development of a golden brown hue. The slides were then rapidly washed in tap water and submitted to a $5 \%$ sodium thiosulphate solution for $3 \mathrm{~min}$. Background staining was by means of diluted Giemsa stain in PBS 3:1, for $10 \mathrm{~min}$. Slides were mounted in balsam.

Immunohistochemistry - Protein Ki-67: deparaffinized sections mounted on glass slides were submitted to the blocking of endogenous avidin by treatment with methanol and 3\% oxygen peroxide. Antigen retrieval was accomplished through micro-wave treatment in citrate buffer at $\mathrm{pH}$ 6.0. Sections were incubated with human anti-Ki-67 antibodies, clone MIB-1, (DAKO, Carpinteria, US) overnight, at $4^{\circ} \mathrm{C}$, in an humidified chamber, under a dilution of 1:100. A biotinilated streptoavidin-peroxidase (Kit LSAB + HRP from DAKO) was used as secundary antibody. The color was developed with $0.06 \%$ 3,3'-diaminobenzidine tetrahydrochloride (DAB) (Sigma) and $0.06 \% \mathrm{H}_{2} \mathrm{O}_{2}$ plus $1 \%$ dimethylsulphoxide (Sigma, St. Louis, US). Sections were counterstained with $1 \%$ methyl-green for $2 \mathrm{~min}$ or Harris hematoxilyn, dehydrated in acetone and mounted in balsam. Control sections in which primary antibody was either omitted or replaced by normal rat serum, were used.

\section{RESULTS}

APO structure - The APO of non-infected B. glabrata appeared as a linear epithelial-like structure, located at the superior-lateral portion of the pericardial sac, near the sacular portion of the kidney. It disclosed the presence of round or cuboidal cells, which were packed together in a few rows (Fig. 1A). The general aspect was rather uniform, changing very little from case to case, even when $S$. mansoni-infected versus non-infected snails were compared. The occasional presence of mitosis was registered. Remnants of S. mansoni sporocysts and developing cercariae were detected in the immediate vicinity or inside the APO tissues. Reactions around these parasitic structures were mild, but sometimes encapsulating reactions were formed around them. The general normal appearance of the APO did not apparently change with the presence of these parasitic lesions (Fig. 1B).

Hemocytes in other sites - In S. mansoni-infected snails hemocytes formed collections around both well preserved and disintegrating sporocysts and immature cercariae. These cells were also seen infiltrating the interstitial tissue, sometimes forming small nodular accumulations. Such changes were most commonly seen in the ovotestis, digestive glands and renal tissue, but also occurred elsewhere. In a few instances the ventricular cavity of the heart appeared filled with hemocytes. When specially searched, collections of hemocytes were most of the times present in the interior of the heart, within the ventricular cavity or infiltrating in between cardiac fibers, even in non-infected snails (Fig. 1C, D). Sometimes the cells lining the inner surface of the vascular spaces seemed to be getting loose and dropping free into the interstitial fluid. This was especially observed in the heart, but was also seen at other areas of the vascular spaces.

The two special tests for revealing mitotic activity Ki67 and AgNOR - gave essentially non-contributory results. The Ki67 protein did not mark any snail structure, but appeared well expressed in developing sporocysts and cercarie (Fig. 2A). The AgNOR marked the nuclei (nucleoli) in cells present within the APO and in several snail tissues, including the hemocytes, and the developing stages of the $S$. mansoni (Fig. 2B).

Under the electron microscope the APO appeared mainly composed by cells packed together, with a definite external membrane that showed interdigitations, thigh adhesions, and even desmosomes in between them. On the side facing the pericardium the lining cells presented numerous filopodia (micro-villi). Large vacuoles containing secretion material were also present in a few cells (Fig. $3 \mathrm{~A})$. On the side where the APO limited with the connective tissue, the epithelial cells rested on a well defined basement membrane (Fig. 3B). The APO cells were rich in organelles such as mitochondria, smooth and rough endoplasmic reticulum, Golgi apparatus, besides a few lysossomes. Alpha glycogen granules were frequently present, sparsely distributed within the cytoplasm. A variable number of hemocytes were present inside and around the APO connective tissue. They exhibited a characteristic star-shape apearance, with several cytoplasmic prolongations, but contained few organelles. No evidences of transitions between the epithelial-like cells and the hemocytes were detected.

Hemocyte counting in lymph - The results are depicted in Fig. 4A. There were no statistically significant differences when the data obtained from infected snails were 

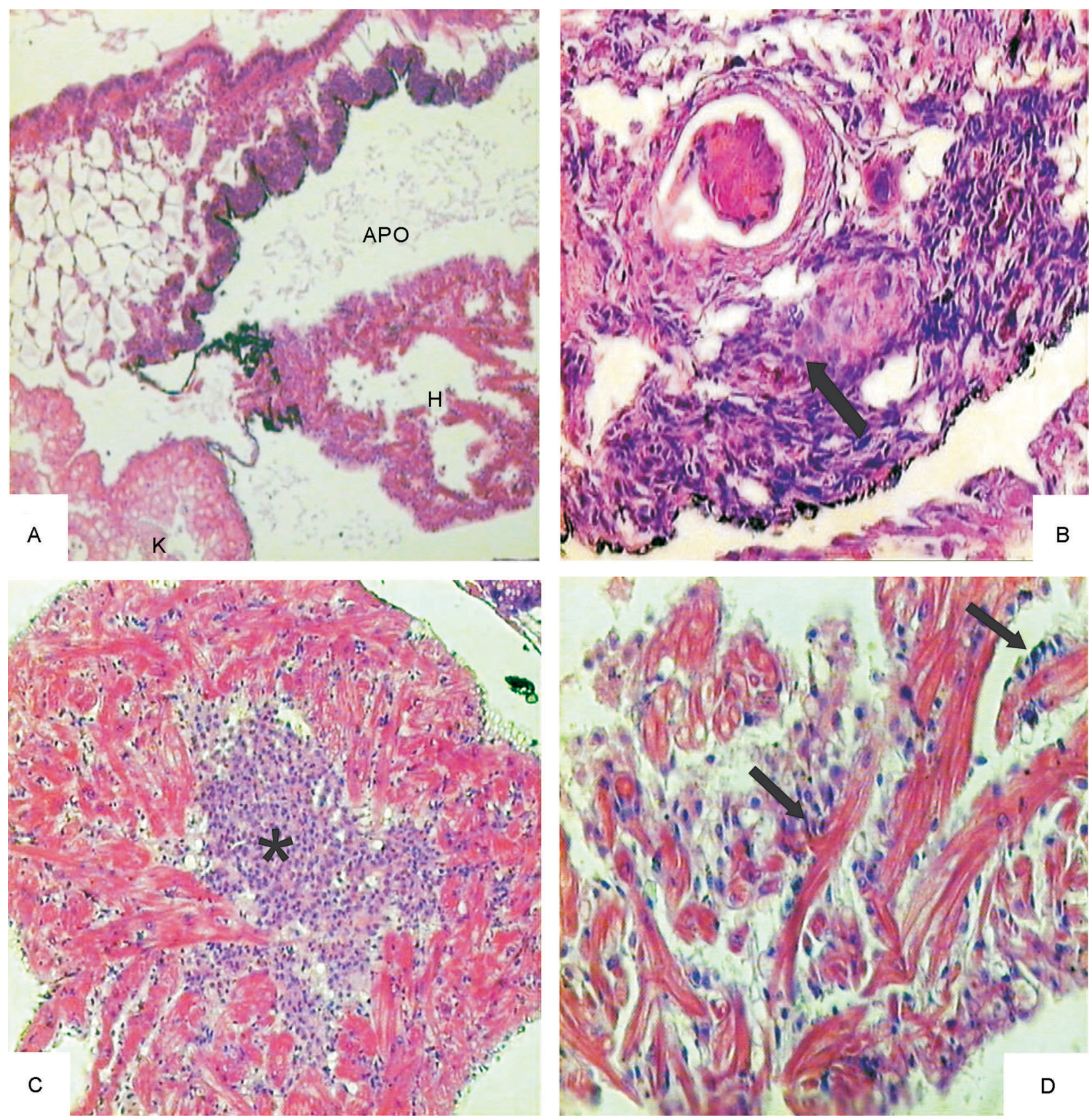

Fig. 1A: the amebocyte-forming organ (APO) appears as a narrow and long band of packed epithelial-like cells, along the pericardial membrane (arrow), at the proximity of the heart $(\mathrm{H})$ and the kidney $(\mathrm{K}), \times 100$; B: disintegrating forms of sporocyts and cercariae found within the APO tissue. An encapsulating reaction was formed around one of them (arrow), but there are not signs of a diffuse reactive change to be noted within the, $\times 200$; $\mathrm{C}$ : a dense collection of amebocytes in the interior of the ventricular cavity of the heart (asterisck) observed in a Schistosoma mansoni-infected Biomphalaria glabrata, $\times 200$; D: a higher magnification of image depicted on C to show details of amebocytes arranged along the endothelial lining of the ventricle, some of them already detached and free within the lymph (arrows). $\times 400$. All picture taken from Hematoxylin \& Eosin stained slides.

compared with those from non-infected ones, except on the 5th day following infection when a slight increase was observed $(\mathrm{P}=0.05)$.

The morphometric analysis of the length and thickness of the APO failed to demonstrate statistically significant differences between infected and non-infected snails (Fig. 4B, C).

\section{DISCUSSION}

The initial studies on the histology of snails postulated that their cells of defense, the hemocytes, had a multicentric origin throughout the endothelial lining of the vascular spaces and the loose connective tissue (Pan 1958, 1963, 1965). However, the current trend considers 


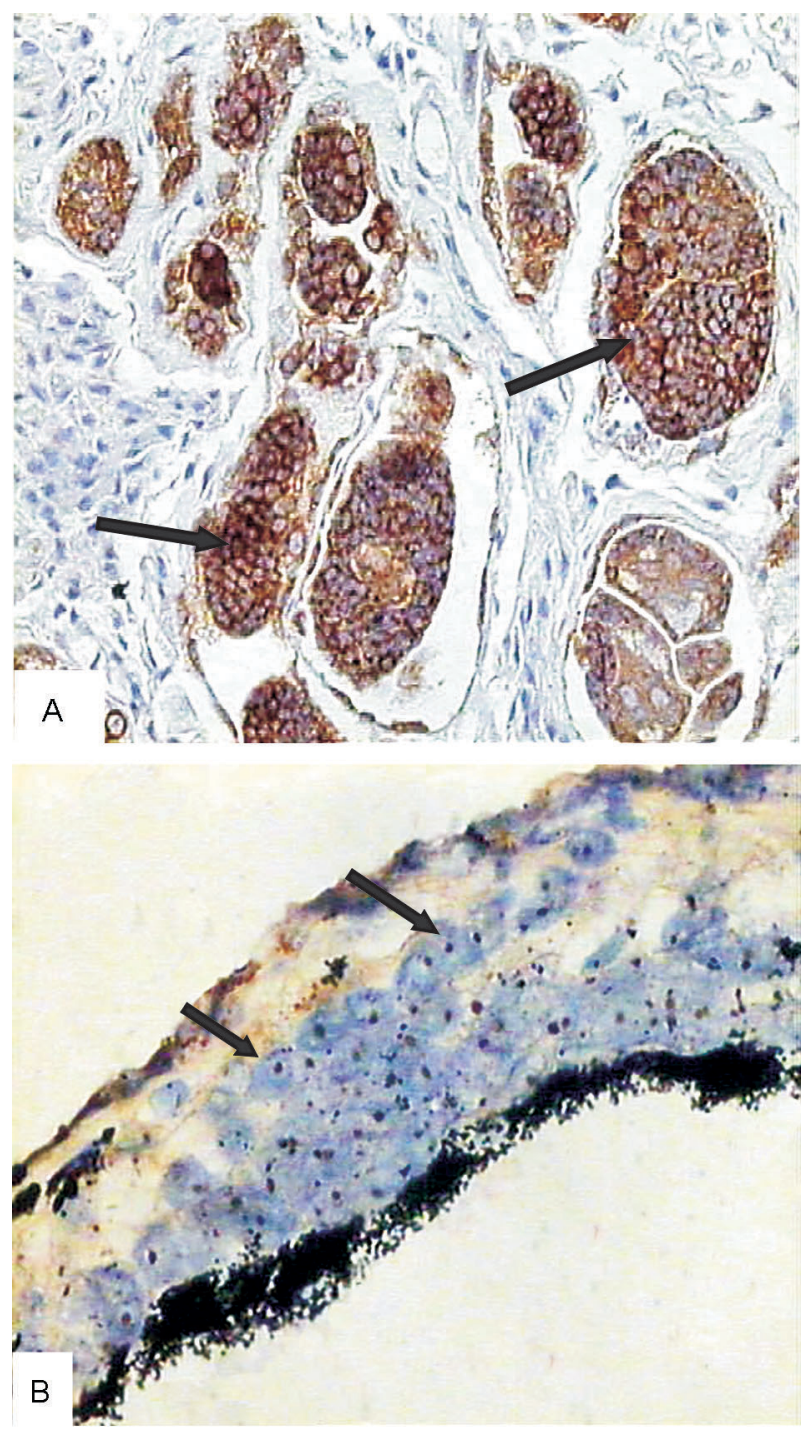

Fig. 2A: staining for the nuclear protein Ki-67 discloses strong nuclear marking in the developmental stages of Schistosoma mansoni, contrasted with its absence in the snail cells (arrows). immunohistochemistry with anti-Ki-67 antibodies, $\times 400$; B: silver impregnation indicative of nucleolar mitotic activity marks numerous amebocytes present within the tissues of $S$. mansoni-infected Biomphalaria glabrata. AgNOR method, $\times 400$.

the mollusk hemocytes as originated from the APO (Lie et al. 1975). This organ is a narrow band of stratified epithelial-like tissue, located within the renoepicardial region. When stimulated it would present cellular hyperplasia and exhibit numerous mitoses while forming specific hematopoietic cells (Sullivan \& Cheng 1984, Sullivan 1990, Sullivan et al. 2004, Sullivan \& Castro 2005). To consider the APO as a central organ, giving origin to all the snail hemocytes, we would have to speculate that a few hemocytes eventually formed in the APO would be able to replicate at peripheral sites. Otherwise the tiny central organ would have to be flooded with cells when a strong response against invading parasites was taking place. On the other hand, the migration of hemocytes from a central point to the several sites of reaction throughout the snail body would be reflected on hemocyte levels in circulating lymph. Such "leukocytosis"-like finding has not so far been demonstrated.

Thus, the present results failed to support the hypothesis of the APO as a central organ for hemocyte production. The B. glabrata APO general structure showed minimal changes during S. mansoni infection, even when disintegrating sporocyts and cercariae were found in its interior. The presence of hyperplasia was not evidenced by histological or morphometric analyses. Data from electron microscopic studies showed the APO to be mainly
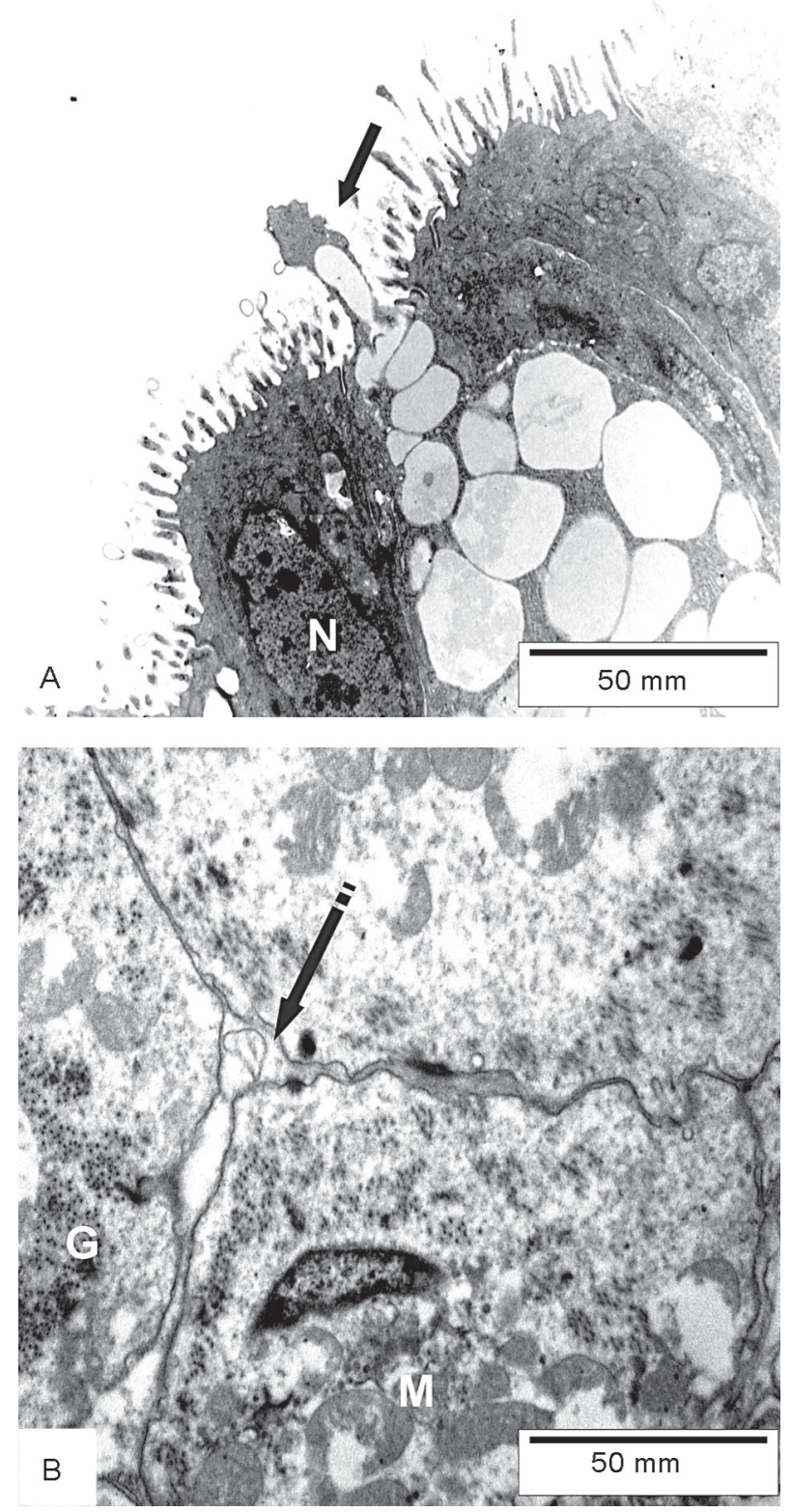

Fig. 3A: secretory cell with large vacuoles in the cytoplasm and an excretory pole. There is an outstanding presence of micro-villi on the free surface of the APO cells; B: APO cells exhibiting interdigitagions between their external membranes (arrows). Mitochondria (M), glycogen granules $(\mathrm{G})$ and nucleus $(\mathrm{N})$ can also be seen. 

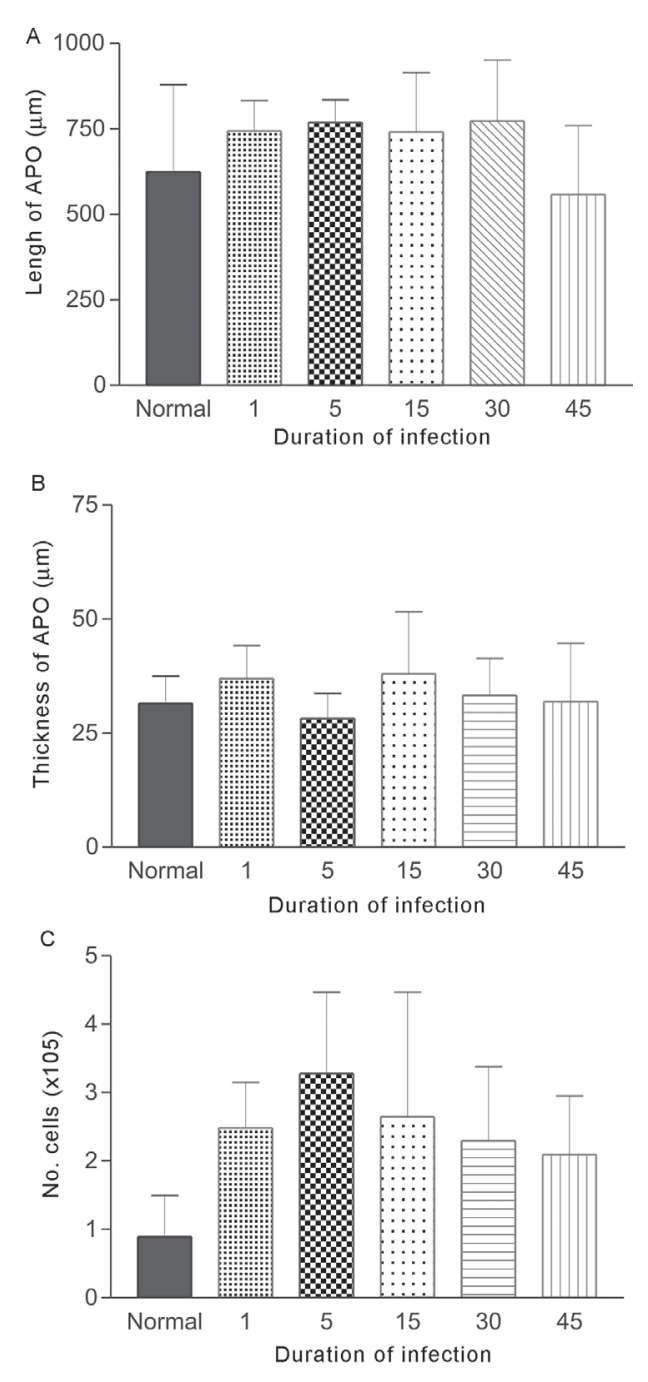

Fig. 4A: hemocyte counts (average) made on fresh hemolymph taken from Schistosoma mansoni-infected Biomphalaria glabrata at different periods of infection. Kruskall-Willis, 95\% confidence interval; B: morphometric analysis of the length of S. mansoniinfected B. glabrata and their non-infected controls. Each bar represents the APO length. Kruskall-Willis, 95\% confidence interval; $\mathrm{C}$ : morphometric analysis of the thickness of $S$. mansoni-infected B. glabrata and their non-infected controls. Each bar represents the APO thickness. Kruskall-Willis, 95\% confidence interval.

composed of epithelial cells, some of them presenting evidences of secretory activity. Also, the presence of micro-villi at the cell free surface, suggests an adaptation to filtration or absorption. No transitional features between the APO-epithelial-like cells and the hemocytes was disclosed. Some experiments have demonstrated that transplantation of an APO from a S. mansoni-resistant snail to a susceptible one, also transfer the resistant character (Sullivan 1990, Sullivan \& Spence 1994). These results need to be examined with caution, before one can be sure that only the APO was transplanted, and not vascular spaces with their endothelial lining. The APO is a microscopic structure, hard to be dissected out. It is even difficult to be found in routine sections taken from a dissected renal-pericardial zone, demanding some variation of serial sectioning.

On the other hand, present findings strongly suggested a multicentric origin for the hemocytes, being entirely in agreement with earlier published findings (Pan 1958, 1963, 1965, Matricon-Gondran 1990, Monteil \& Matricon-Gondran 1991, Sminia 1972, 1974). The endothelial lining of the vascular spaces and the loose connective tissue showed reactions in S. mansoni-infected snails suggestive of focal proliferation and hemocyte differentiation. Also, the presence of nuclear changes and even mitoses in hemocytes indicated their capacity for local proliferation. The ventricular cavity of the heart appeared as a frequent and impressive site of hemocyte concentration. At that site the transitional features between fixed endothelial lining cells and free hemocytes could be traced. One is induced to speculate that hemocytes being inside the ventricular cavity of the heart seem a good adaptation for an emergency delivery of those cells to any part of the body. However, it would be unnecessary, since hemocytes can be formed anywhere from the snails tissue, including the APO.

The formation of blood cells from endothelial line is reminiscent of early evolutionary stages, when mesenchymal cells delimit vascular spaces to form vessels and blood cells throughout the developing organism.

\section{REFERENCES}

Jeong KH, Lie KJ, Heyneman D.1983. The ultrastructure of the amebocyte-producing organ in Biomphalaria glabrata. Dev Com Immunol 7: 217-228.

Lie KJ, Heyneman D 1975. Studies on resistance in snails: a specific tissue reaction to Echinostoma lindoense in Biomphalaria glabrata. Int J Parasitol 15: 621-626.

Matricon-Gondran M 1990 The site of ultrafiltration in the kidney sac of the pulmonate gastropod Biomphalaria glabrata. Tissue Cell 22: 911-923.

Monteil JF, Matricon-Gondran M 1991. Hemocyte production in trematode-infected Lymnaea truncatula. Parasitol Res 77: 491-497.

Pan CT 1958. The general histology and topographic microanatomy of Australorbis glabrata. Bull Mus Comp Zool 119: 237-99.

Pan CT 1963. Generalized and focal tissue responses in the snail, Australorbis glabratus, infected with Schistosoma mansoni. An NY Acad Sci 113: 475-485.

Pan CT 1965. Studies on the host-parasite relationship between Schistosoma mansoni and the snail Australorbis glabrata. Am J Trop Med Hyg 14: 931-975.

Sminia T 1972. Structure and function of blood and connective tissue cells of the fresh water pulmonate Lymnaea stagnalis studied by electron microscopy and enzyme histochemistry. Z Zellforsch 130: 497-526.

Sminia T 1974. Haematopoiesis in the freshwater snail Lymnaea stagnalis studied by electron microscopy and autoradiography. Cell Tiss Res 150: 443-454.

Sullivan JT 1990. Long-term survival of heterotopic allografts of the amebocyte-producing organ in Biomphalaria glabrata (Mollusca: Pulmonata). Trans Am Microsc Soc 109: 52-60. 
Sullivan JT, Castro L 2005. Mitotic arrest and toxicity in Biomphalaria glabrata (Mollusca: Pulmonata) exposed to colchicine. J Invertebr Pathol 90: 32-38.

Sullivan JT, Cheng TC 1984. Mitotic responses of the anterior pericardial wall of Biomphalaria glabrata (Molusca) subjected to challeng. J Invertebr Pathol 44: 114-116.
Sullivan JT, Spence JV 1994. Transfer of resistance to Schistosoma mansoni in Biomphalaria glabrata by allografts of amoebocyte-producing organ. J Parasitol 80: 449-453.

Sullivan JT, Pikios SS, Alonzo AQ 2004. Mitotic responses to extracts of miracidia and cercarie of Schistosoma mansoni in the amebocyte-producing organ of the snail intermediate host Biomphalaria glabrata. J Parasitol 90: 92-96. 